\title{
Correlates of Medical Nutrition Therapy and Cardiovascular Outcomes in Youth with Type 1 Diabetes
}

\author{
Natalie S. The, Ph.D. ${ }^{a}$, Jamie L. Crandell, Ph.D. ${ }^{b}$, Joan Thomas, M.S. ${ }^{a}$, Sarah C. Couch, \\ Ph.D. ${ }^{c}$, Amy S. Shah, M.D. ${ }^{d}$, David M Maahs, M.D. Ph.D. ${ }^{e}$, Dana Dabelea, M.D. Ph.D. ${ }^{f}$, \\ Santica M. Marcovina, Ph.D. Sc.D. ${ }^{g}$, Ralph B. D'Agostino Jr., Ph.D. ${ }^{h}$, and Elizabeth J. \\ Mayer-Davis, Ph.D. ${ }^{a}$ for the Search for Diabetes in Youth Study Group \\ aDepartment of Nutrition, Gillings School of Global Public Health, University of North Carolina, \\ Chapel Hill, NC, 27599, USA \\ bDepartment of Biostatistics, Gillings School of Global Public Health, School of Nursing, \\ University of North Carolina, Chapel Hill, NC, 27599, USA \\ 'Department of Nutritional Sciences, College of Allied Health, University of Cincinnati, Cincinnati, \\ $\mathrm{OH}, 45221$, USA \\ ${ }^{\mathrm{d} D i v i s i o n}$ of Endocrinology, Cincinnati Children's Hospital Medical Center, Cincinnati, OH, 45229, \\ USA
}

eBarbara Davis Center and Department of Pediatrics, School of Medicine, University of Colorado Denver, Aurora, CO, 80045, USA

fDepartment of Epidemiology, Colorado School of Public Health, University of Colorado Denver, Aurora, CO, 80045, USA

9Northwest Metabolism and Diabetes Research Laboratories, University of Washington, Seattle, Washington, 98109, USA

hDepartment of Biostatistical Sciences, School of Medicine, Wake Forest University, WinstonSalem, NC, 27157, USA

\section{Abstract}

Objective-To examine whether the types of medical nutrition therapies (MNTs) taught to and used by youth with type 1 diabetes (T1D) varies by socio-demographic characteristics and cardiovascular (CVD) risk factors

Design-Cross-sectional study

(C) 2013 Society for Nutrition Education. Published by Elsevier Inc. All rights reserved.

Corresponding Author: Elizabeth J Mayer-Davis, PhD, 2211 McGavran-Greenberg Hall, Campus Box 7461, The University of North Carolina at Chapel, Hill, Chapel Hill, NC 27599., Phone (919) 966-1991; Facsimile (919) 966-7216; ejmayer-davis@unc.edu.

Publisher's Disclaimer: This is a PDF file of an unedited manuscript that has been accepted for publication. As a service to our customers we are providing this early version of the manuscript. The manuscript will undergo copyediting, typesetting, and review of the resulting proof before it is published in its final citable form. Please note that during the production process errors may be discovered which could affect the content, and all legal disclaimers that apply to the journal pertain. 
Setting-The SEARCH for Diabetes in Youth study is a population-based cohort of individuals with clinical diagnosed diabetes

Participants-1,191 individuals with T1D

Main Outcome Measures-Types of MNTs and frequency of use

Analysis-Bivariate analysis and multivariate linear regression $(P<0.05)$

Results-More race/ethnic minorities (vs. whites), individuals with parents <high school education (vs. ๖high school), and overweight/obese (vs. underweight/normal weight) were taught additional MNTs. For underweight/normal weight individuals exclusively taught carbohydrate counting, those who used this approach "often" had lower A1c (8.6 vs. 8.9\%) and triglycerides (73.5 vs. $84.1 \mathrm{mg} / \mathrm{dL}$ ) than those who used it "sometimes/never." "Often" use of additional MNTs beyond carbohydrate counting was not associated with better mean values for CVD risk factors.

Conclusions and Implications-In individuals with T1D, race/ethnic minorities, individuals with parents < high school education, and overweight/obese individuals are taught more MNTs. Further research is needed to understand the effectiveness of the various MNTs on CVD risk factors, and identify how to translate nutrition knowledge to behavior and metabolic status.

\section{Keywords}

medical nutrition therapy; type 1 diabetes; cardiovascular risk

\section{Introduction}

Dietary behavior is an important, modifiable patient-associated factor for optimizing health among youth with diabetes. ${ }^{1}$ The primary goals of medical nutrition therapy (MNT) for youth with diabetes is to optimize glycemic control, minimize acute and chronic complications, and achieve normal growth and development through dietary choices that are attainable and sustainable. ${ }^{2}$ They are advised to follow a dietary pattern that emphasizes consumption of whole grains, fruits, vegetables, and low fat milk, and limits consumption of saturated and trans fats. ${ }^{1}$ Carbohydrate monitoring is also emphasized given that carbohydrates have the greatest influence on postprandial blood glucose levels. ${ }^{3}$ There are numerous medical nutrition therapies (MNTs) available to help individuals adhere to a healthy diet including nutrient-based approaches, glycemic index, and food-based approaches. However, less is known about the frequency of which MNTs are being taught to and used by youth with T1D.

Previous research suggests that youth with diabetes are not meeting recommended dietary guidelines ${ }^{4}$ and are consuming more total and saturated fat than children without diabetes; 5,6 These findings are concerning given that $15 \%$ of youth with T1D have $\geq 2$ traditional CVD risk factors present in addition to glucose intolerance compared to $6 \%$ in youth without diabetes. ${ }^{7}$ Further, CVD risk factors have been shown to track from childhood to adulthood. ${ }^{8}$ Thus, a detailed exploration of the MNTs taught to and used by youth with T1D is needed. 
The objectives of this study were two-fold. The first aim was to examine the frequency of the types of MNTs being taught to youth with T1D, hypothesizing that this would vary by socio-demographic factors and by weight status. The second aim was to explore the crosssectional associations of the frequency of specific MNT use with several CVD risk factors, hypothesizing that these associations vary by weight status.

\section{Methods}

A detailed description of SEARCH study methods has been published elsewhere. ${ }^{9}$ Briefly, the SEARCH Study began conducting population-based ascertainment of youth $(<20$ years of age) newly diagnosed diabetes starting in 2002 and continuing through the present. SEARCH recruited participants from four geographically defined populations in Ohio, Colorado, South Carolina and Washington; Indian Health Service beneficiaries from four American Indian populations, and enrollees in several managed health care plans in California and Hawaii. The study was reviewed and approved by the local Institutional Review Board(s) that had jurisdiction over the local study populations. At the time of the initial SEARCH study visit, informed consent was obtained, physical measurements and blood samples (after an overnight fast) were obtained from metabolically stable participants, and questionnaires were administered. Participants were invited for follow-up visits at approximately 24 and 60 months after their baseline visit.

\section{Measures}

Information on MNT was assessed as part of the SEARCH Quality of Care Survey administered at the 24 and 60 month follow-up visits. Two versions of the survey were used, one for participants age $\geq 18$ years at the time of the survey to complete on their own, and one for participants age $<18$ years that was completed by the participant's parent or guardian (available in English or in Spanish). Participants reported ("yes," "no," or "don't know") if they were ever taught the following MNTs by their health care providers: count carbohydrates, track calories, track fat grams, glycemic index, and food exchanges. All participants who reported "don't know" were classified as missing. If participants reported "yes" to learning any of the MNTs, they were then asked to report whether they currently used that approach "often," "sometimes" or "never."

At each study visit, standardized anthropometric measurements were made, and height and weight measurements were used to calculate body mass index (BMI, $\mathrm{kg} / \mathrm{m}^{2}$ ). Age- and gender specific BMI z-scores were derived using CDC standards. ${ }^{10}$ Weight status was categorized as underweight/normal weight $\left(\mathrm{BMI}<85^{\text {th }}\right.$ percentile for age and sex) and overweight/obese (BMI $\geq 85^{\text {th }}$ percentile for age and sex). Blood pressure measurements (systolic blood pressure [SBP] and diastolic blood pressure [DBP]) were obtained using a portable manometer and conducted after the patient had been sitting for at least 5 minutes. For the measurement of A1c and lipids (total cholesterol, low-density lipoprotein [LDL]cholesterol, high-density lipoprotein [HDL]-cholesterol, and triglycerides), blood samples (after an overnight fast) were obtained at each visit under conditions of metabolic stability, defined as no episode of diabetic ketoacidosis during the previous month. Specimens were processed locally and shipped within 24 hours to the central laboratory (Northwest Lipid 
Metabolism and Diabetes Research Laboratories, University of Washington, Seattle). Details on methods used to measure A1c, lipid profile and blood pressure have been published previously. ${ }^{9,11}$

Self-reported race and ethnicity were collected based on questions modeled after 2000 US Census and categorized as non-Hispanic white, African American, Hispanic, and "Other." 12 Parental education was based on the highest educational level attained by either parent and dichotomized as $\leq$ high school or $>$ high school. For participants ages $\geq 10$ years, SEARCH ascertained physical activity status via questionnaire. ${ }^{13}$ Moderate to vigorous physical activity was defined as $\geq 3$ days/week of any activity that either tones or makes one sweat.

\section{Subject Inclusion}

This analysis includes data from individuals with T1D (defined by physicians' report of "type 1", "type 1a", or "type 1b" diabetes) diagnosed in 2002-2005 who participated in the SEARCH baseline examination $(n=2,326)$ and completed follow-up visits at either 24months or 60-months after their baseline visit. By September 29, 2010 (the close of the 24and 60-month data collection period), 1,572 had attended the 24- and/or 60-month follow-up visit. Mainly because SEARCH began administering the Quality of Care survey after the initiation of the follow-up data collection period, 1,295 (82\%) completed the series of questions about MNT. Participants who had self-reported taking hypertensive and/or lipidlowering medications were also excluded $(\mathrm{n}=66[5 \%])$, as these individuals may represent a unique group of individuals with diabetes who are being medically treated more aggressively than representative of other youth with T1D. Of the remaining 1,229 eligible participants, $97 \%$ were taught carbohydrate counting by their healthcare provider. Given the strong emphasis on carbohydrate monitoring for individuals with T1D, the 38 participants who were not taught carbohydrate counting were excluded as they are not representative of individuals with T1D. Thus, there were 1,191 participants included in this analysis and all were taught carbohydrate counting. For individuals who had data from both 24- and 60month follow-up visits $(\mathrm{n}=299$ [23\%]), we used data from the last visit (e.g. 60-month follow-up).

\section{Statistical Analyses}

Statistical analyses were performed using SAS software (version 9.2; SAS Institute, Cary, NC). Comparisons of mean values and percentages by participant characteristics were examined using ANOVA and $\chi^{2}$ tests. Multivariate linear regression modeling was used to examine the association between learning and using MNT approaches with CVD risk factors (A1C, SBP, DBP, total cholesterol, HDL-cholesterol, LDL-cholesterol, and triglycerides), with statistical significance established at $P<0.05$. To determine whether the association between being taught and using MNT approaches with CVD risk factors would vary by BMI category by use of interaction terms (BMI category* MNT) and Wald tests were used to examine effect modification. A relaxed criterion $(P<0.15)$ for effect modification was used due to the descriptive and exploratory nature of these analyses and observed significant effect modification by BMI. Thus, for comparability, all models were stratified by BMI category. Models were adjusted for race/ethnicity, gender, parental education, study site, age at visit, diabetes duration, and insulin regimen. To facilitate the interpretation of $\beta$ 
coefficients from multivariate models, mean values were estimated for CVD risk factors by various MNT approaches, while other covariates were set to the sample means. A similar analysis was repeated among youth $\geq 10$ years of age, adjusting for physical activity.

\section{Results}

Descriptive characteristics of study participants at the follow-up visit that provided MNT data (either 24- or 60-month) are provided in Table 1. Half of participants were female, a majority of participants were non-Hispanic white (78\%), and the mean age was 14.1 years.

Among participants, $25 \%$ of participants were taught to track fat grams, $22 \%$ to track calories, 37\% to choose low glycemic index foods, and 33\% to use food exchanges (Figure 1), with variation by race/ethnicity, parental education, weight status, and study site. Race/ ethnic differences persisted after adjustment for BMI z-score, with minorities being more likely to be taught to track fat grams (Black: 40\%; Hispanic: 46\%; Other: 38\%), track calories (Black: 46\%; Hispanic: 48\%), and food exchanges (Black: 63\%; Hispanic: 56\%; Other: 58\%) than non-Hispanic whites (fat grams: 35\%; calories: 33\%; exchanges: 50\%). Additionally, learning food exchanges varied by parental education after adjustment for BMI z-score, with a higher percentage of individuals with parents shigh school education learning food exchanges.

Nearly half $(43 \%)$ of participants were exclusively taught carbohydrate counting. One-fourth of individuals were taught 1 additional MNT, 13\% taught two, 7\% taught three, and 11\% taught four-MNTs s beyond carbohydrate counting. The most commonly taught 3-way combination taught to individuals with T1D was carbohydrate counting, glycemic index and food exchanges (21\%) (Table 2). Overweight/obese individuals were more likely to be taught a combination of 3-, 4-, (10\% vs. $6 \%)$, and 5- MNTs (14\% vs. $9 \%)$ than underweight/ normal weight individuals (all $P<0.05$ ).

For individuals who were exclusively taught carbohydrate counting, estimated mean values and their $95 \%$ confidence intervals (CI) for CVD risk factors by how frequent they used the approach are shown in Table 3, according to BMI category. Among normal weight/ underweight individuals, those who used this method "often" had significantly lower mean values of triglycerides and A1c compared to those who used this method "sometimes/ never." Similarly, those who used carbohydrate counting "often" had lower DBP and total cholesterol compared to those who used this method "sometimes/never," but this was borderline significant (DBP: $P=0.05$; total cholesterol: $P=0.06$ ). In contrast, among overweight/obese individuals who were exclusively taught carbohydrate counting, those who used this method "often" had similar mean values for CVD risk factors as those who used this method "sometimes/never." Among youth $\geq 10$ years of age for whom physical activity was assessed, additional adjustment for physical activity resulted in similar findings (results not shown).

For individuals who were taught carbohydrate counting and used this approach "often," we evaluated whether the addition and use of other MNTs was associated with CVD risk factors, stratified by BMI category (Table 4). Among underweight/normal weight 
individuals, those who "often" used an additional MNT beyond carbohydrate counting had lower mean SBP than individuals who were exclusively taught carbohydrate counting. Among overweight/obese individuals, those who "often" used an additional MNT beyond carbohydrate counting had higher mean LDL- and total cholesterol than individuals who were exclusively taught carbohydrate counting. In subsequent analyses, the addition and use of glycemic index (beyond exclusive and "often" use of carbohydrate counting) was not associated with A1c. Individuals who used the method "often (mean = 8.7, CI = 8.2-9.1)" or "sometimes/never (mean $=8.8, \mathrm{CI}=8.4-9.1$ )" had similar mean A1c compared to individuals who were exclusively taught and "often" used carbohydrate counting (mean = $8.6, \mathrm{CI}=8.2-9.0)$.

\section{Discussion}

In this SEARCH study of individuals with T1D, race/ethnic minorities (versus non-Hispanic whites) and individuals with parents who had $<$ high school education (versus $\geq$ high school education) were more likely to be taught a combination of additional MNTs beyond carbohydrate counting. These differences were not influenced by weight status. Additional MNTs may be the direct response by health care providers to increased occurrence of adverse metabolic profile among these demographic subgroups. ${ }^{11,14}$ It is also possible that race/ethnic minorities and individuals of low socio-economic status (SES) have or are perceived as having low-dietary adherence ${ }^{15,16}$ or have lower quality ${ }^{17}$ resulting in health care providers teaching additional MNTs to populations that are traditionally at risk. Alternatively, there may be differences in type of health care, health care provider bias related to the types of MNT delivered to certain populations, or over-reporting of the types of MNTs learned. Because race/ethnic minorities and individuals of low SES commonly have poor glycemic control, ${ }^{18}$ adverse CVD risk profiles, ${ }^{11}$ and are especially vulnerable to diabetes complications, understanding these discrepancies is critical, including the comparative effectiveness of different MNTs for various dietary and metabolic goals across various population subgroups.

The importance of findings from this study lies in the potential that MNT (in conjunction with other components of diabetes care) can improve metabolic outcomes, ${ }^{19}$ which is especially significant for individuals with T1D since a higher percentage of them have $\geq 2$ CVD risk factors later in life than those without diabetes. ${ }^{11}$ For normal weight/underweight individuals, we observed that individuals who were exclusively taught carbohydrate counting and used this approach "often" had lower mean levels of A1c and triglycerides than those who used the method "sometimes/never." The addition and "often" use of other MNTs compared to exclusive carbohydrate counting use was not associated with improved mean values for CVD risk factors. However, it is possible that individuals who learned additional MNTs may have been taught these approaches because carbohydrate counting was not attainable or sustainable for these individuals. As a result, persons learning multiple MNTs may be those at greatest risk for dietary non-adherence and CVD risk. Alternatively, it may be that individuals who "often" used an additional MNT did improve their CVD risk profile to that of individuals who exclusively used carbohydrate counting. These changes would not be captured in this cross-sectional analysis. The longitudinal relationships 
between being taught and using MNTs, dietary consumption, and CVD risk factors are an area of great research potential.

All individuals with T1D need to coordinate diet and insulin regimen for day-to-day glycemic control; however, overweight and obese individuals with T1D have the added burden of weight management. ${ }^{2}$ Interestingly, there were significant differences in the association of MNTs learned and their frequency of use with CVD risk factors. Overweight and obese individuals were more likely to learn a combination of MNTs than normal weight and underweight individuals. However, "often" use of exclusively learned carbohydrate counting and the addition of additional MNTs were not associated with improved metabolic outcomes in the cross-sectional analyses. While prospective studies are needed, these findings emphasize the importance of identifying barriers to dietary adherence and translating nutrition knowledge to behavior.

There are some limitations, as well as strengths, to this study. An important limitation of this research is the use of self-report related to which MNTs were taught and how often each approach was used by the participant. Some individuals may respond to questionnaire items in ways they perceive to be socially desirable, ${ }^{20}$ which may result in biased reporting. Misclassification of the use of MNTs may explain null associations between uses of a combination of MNTs and CVD risk factors. Second, it is possible that participation in the SEARCH follow-up visit cohort may have introduced selection bias. Individuals who participated may be more likely to be concerned about their health, and thus may have been taught more MNTs and/or use these approaches more "often" than their non-participating peers. If this were true, then individuals with T1D in the total population may be taught fewer MNTs and use these approaches less "often." Third, parents of youth < 18 years of age completed the survey about what was taught and actual behaviors. These parental reports may be less accurate for older youth who are more responsible for their own care, including MNT. Finally, this investigation used a cross-sectional design, and thus the temporal relationship between learning and use of MNTs with cardiometabolic risk cannot be established. Despite these limitations, this study has important strengths. The SEARCH study utilized observational data from a large, diverse contemporary sample of youth and young adults with T1D. SEARCH includes measures of key CVD risk factors and critical information regarding which MNTs are taught and how often individuals use these approaches, thus, allowing us to explore if certain combinations of MNTs provide a greater influence on metabolic outcomes than other approaches.

\section{Implications for Research and Practice}

This study has several potential implications. First, there is significant variation in MNTs taught and frequency of their use among individuals with T1D. It may be that individuals who have lower adherence to a healthy diet are taught more MNT approaches. A better identification of barriers to dietary adherence and translation of nutrition knowledge to behavior is needed. Further, research should examine whether patient-centered counseling and motivational interviewing improves dietary adherence. Findings also suggest that adherence to MNT may improve metabolic outcomes. Underweight/normal weight individuals who exclusively learn carbohydrate counting and use this approach "often" have 
improved metabolic status than those who use this method "sometimes/never." Conversely, learning additional MNTs beyond carbohydrate counting appears to have limited benefit on CVD risk profile; however, further research is needed to elucidate the mediators of improved metabolic status. Continued efforts should be made to identify effective dietary counseling approaches for individuals with T1D.

\section{Acknowledgments}

The SEARCH for Diabetes in Youth Study and the SEARCH Nutrition Ancillary Study (SNAS) is indebted to the many youth, their families, and their health care providers whose participation made this study possible. The SEARCH for Diabetes in Youth is funded by the Centers for Disease Control and Prevention (CDC; PA numbers 00097, DP-05-069, and DP-10-001) and supported by the National Institute of Diabetes and Digestive and Kidney Diseases (NIDDK). Site Contract Numbers: Kaiser Permanente Southern California (U48/CCU919219, U01 DP000246, and U18DP002714), University of Colorado Denver (U48/CCU819241-3, U01 DP000247, and U18DP000247-06A1), Kuakini Medical Center (U58CCU919256 and U01 DP000245), Children's Hospital Medical Center (Cincinnati) (U48/CCU519239, U01 DP000248, and 1U18DP002709), University of North Carolina at Chapel Hill (U48/CCU419249, U01 DP000254, and U18DP002708-01), University of Washington School of Medicine (U58/CCU019235-4, U01 DP000244, and U18DP002710-01), Wake Forest University School of Medicine (U48/CCU919219, U01 DP000250, and 200-2010-35171). SNAS is funded by NIH/NIDDK R01 DK077949 (Mayer-Davis, PI).

The authors wish to acknowledge the involvement of General Clinical Research Centers at the South Carolina Clinical \& Translational Research Institute, at the Medical University of South Carolina (NIH/NCRR Grant number UL1RR029882); Children's Hospital and Regional Medical Center (Grant Number M01RR00037); Colorado Pediatric General Clinical Research Center (Grant Number M01 RR00069) and the Barbara Davis Center at the University of Colorado at Denver (DERC NIH P30 DK57516); and the Institutional Clinical and Translational Science Award, NIH/NCRR at the University of Cincinnati (Grant Number 1UL1RR026314-01).

The contents of this paper are solely the responsibility of the authors and do not necessarily represent the official views of the CDC and the NIDDK.

\section{References}

1. Bantle JP, Wylie-Rosett J, Albright AL, et al. Nutrition recommendations and interventions for diabetes: a position statement of the American Diabetes Association. Diabetes Care. Jan; 2008 31(1):S61-78. [PubMed: 18165339]

2. Smart C, Aslander-van Vliet E, Waldron S. Nutritional management in children and adolescents with diabetes. Pediatr Diabetes. Sep; 2009 10(12):100-117. [PubMed: 19754622]

3. Sheard NF, Clark NG, Brand-Miller JC, et al. Dietary carbohydrate (amount and type) in the prevention and management of diabetes: a statement by the american diabetes association. Diabetes Care. Sep; 2004 27(9):2266-2271. [PubMed: 15333500]

4. Mayer-Davis EJ, Nichols M, Liese AD, et al. Dietary intake among youth with diabetes: the SEARCH for Diabetes in Youth Study. J Am Diet Assoc. May; 2006 106(5):689-697. [PubMed: 16647326]

5. Helgeson VS, Viccaro L, Becker D, Escobar O, Siminerio L. Diet of adolescents with and without diabetes: Trading candy for potato chips? Diabetes Care. May; 2006 29(5):982-987. [PubMed: 16644624]

6. Overby NC, Flaaten V, Veierod MB, et al. Children and adolescents with type 1 diabetes eat a more atherosclerosis-prone diet than healthy control subjects. Diabetologia. Feb; 2007 50(2):307-316. [PubMed: 17136391]

7. Duncan GE, Li SM, Zhou XH. Prevalence and trends of a metabolic syndrome phenotype among u.s. Adolescents, 1999-2000. Diabetes Care. Oct; 2004 27(10):2438-2443. [PubMed: 15451913]

8. Nicklas TA, von Duvillard SP, Berenson GS. Tracking of serum lipids and lipoproteins from childhood to dyslipidemia in adults: the Bogalusa Heart Study. International journal of sports medicine. May; 2002 23(1):S39-43. [PubMed: 12012261]

9. SEARCH for Diabetes in Youth: a multicenter study of the prevalence incidence and classification of diabetes mellitus in youth. Control Clin Trials. Oct; 2004 25(5):458-471. [PubMed: 15465616] 
10. Kuczmarski, RJ.; Ogden, CL.; Grummer-Strawn, LM., et al. Adv Data. Jun 8. 2000 CDC growth charts: United States; p. 1p. 27

11. Rodriguez BL, Fujimoto WY, Mayer-Davis EJ, et al. Prevalence of cardiovascular disease risk factors in U.S. children and adolescents with diabetes: the SEARCH for diabetes in youth study. Diabetes Care. Aug; 2006 29(8):1891-1896. [PubMed: 16873798]

12. Mayer-Davis EJ, Bell RA, Dabelea D, et al. The many faces of diabetes in American youth: type 1 and type 2 diabetes in five race and ethnic populations: the SEARCH for Diabetes in Youth Study. Diabetes Care. Mar; 2009 32(2):S99-101. [PubMed: 19246580]

13. Kann L, Kinchen SA, Williams BI, et al. Youth risk behavior surveillance--United States, 1999. MMWR CDC surveillance summaries: Morbidity and mortality weekly report CDC surveillance summaries/Centers for Disease Control. Jun 9; 2000 49(5):1-32. [PubMed: 12412614]

14. Petitti DB, Klingensmith GJ, Bell RA, et al. Glycemic control in youth with diabetes: the SEARCH for diabetes in Youth Study. J Pediatr. Nov; 2009 155(5):668-672. e661-663. [PubMed: 19643434]

15. Bortsov A, Liese AD, Bell RA, et al. Correlates of Dietary Intake in Youth with Diabetes: Results from the SEARCH for Diabetes in Youth Study. J Nutr Educ Behav. Jan 26.2011

16. Storey ML, Forshee RA, Anderson PA. Associations of adequate intake of calcium with diet, beverage consumption, and demographic characteristics among children and adolescents. J Am Coll Nutr. Feb; 2004 23(1):18-33. [PubMed: 14963050]

17. Hiza HA, Casavale KO, Guenther PM, Davis CA. Diet quality of americans differs by age, sex, race/ethnicity, income, and education level. Journal of the Academy of Nutrition and Dietetics. Feb; 2013 113(2):297-306. [PubMed: 23168270]

18. Mayer-Davis EJ, Beyer J, Bell RA, et al. Diabetes in African American youth: prevalence, incidence, and clinical characteristics: the SEARCH for Diabetes in Youth Study. Diabetes Care. Mar; 2009 32(2):S112-122. [PubMed: 19246576]

19. Mehta SN, Volkening LK, Anderson BJ, et al. Dietary behaviors predict glycemic control in youth with type 1 diabetes. Diabetes Care. Jul; 2008 31(7):1318-1320. [PubMed: 18390798]

20. Hebert JR, Peterson KE, Hurley TG, et al. The effect of social desirability trait on self-reported dietary measures among multi-ethnic female health center employees. Ann Epidemiol. Aug; 2001 11(6):417-427. [PubMed: 11454501] 


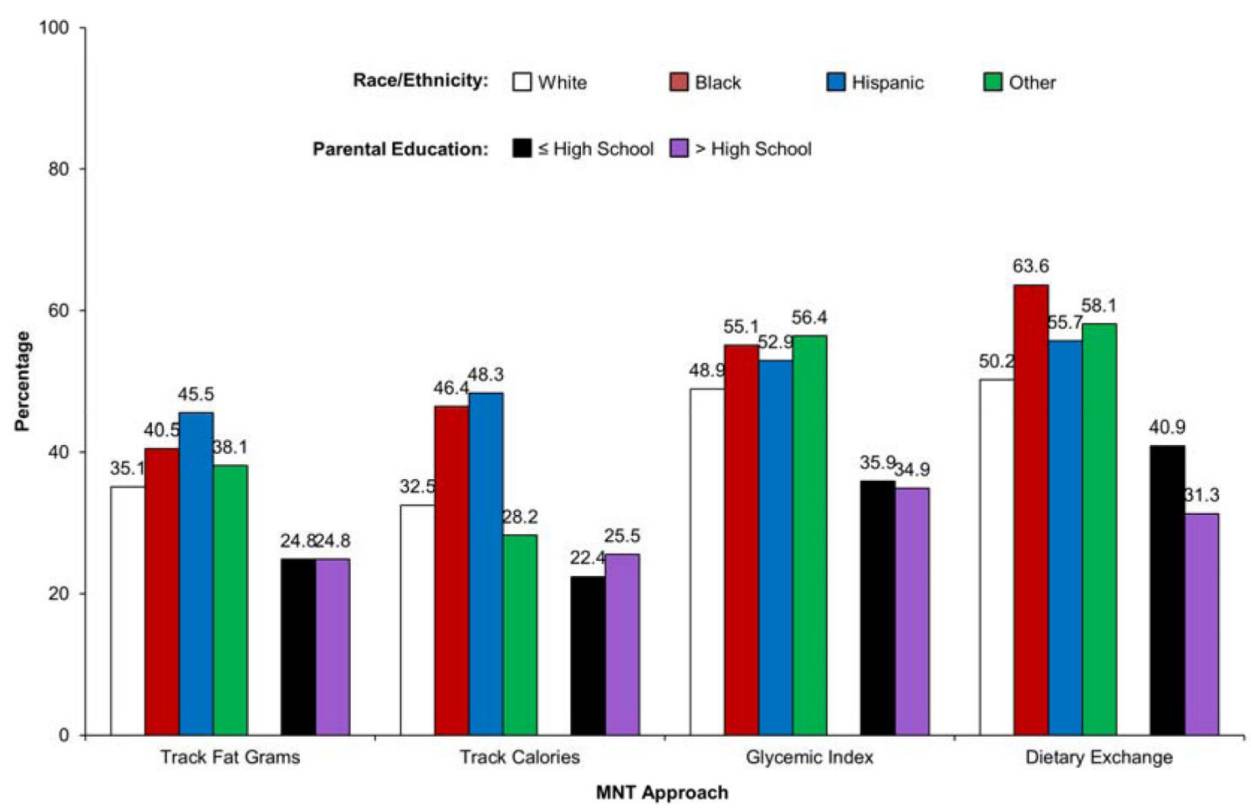

Figure 1. Proportion a of Individuals Reporting being Taught Specific MNT Approaches by Race/Ethnicityb and Parental Education ${ }^{c}(n=1,191)$

adjusted for BMI z-score.

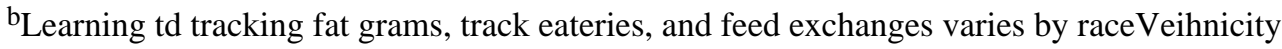
$(\mathrm{p}<0.05)$.

${ }^{c}$ Learning food exchanges varies by parental education $(\mathrm{p}<0.05)$. 
Table 1

Characteristics of Individuals with Type 1 Diabetes Diagnosed in 2002-2005 (n=1,191)

\begin{tabular}{|c|c|c|}
\hline Characteristic & $\mathbf{n}$ & $\%^{a}$ or mean $(\mathrm{SD})$ \\
\hline Age at visit (years) & 1,191 & $14.1(4.5)$ \\
\hline \multicolumn{3}{|l|}{ Gender } \\
\hline Female & 597 & 50.1 \\
\hline Male & 594 & 49.9 \\
\hline \multicolumn{3}{|l|}{ Race } \\
\hline non-Hispanic White & 925 & 77.7 \\
\hline African American & 106 & 8.9 \\
\hline Hispanic & 105 & 8.8 \\
\hline Other & 55 & 4.6 \\
\hline \multicolumn{3}{|l|}{ Parental Education } \\
\hline$\leq$ High School & 196 & 16.6 \\
\hline$>$ High School & 986 & 83.4 \\
\hline Missing & 12 & \\
\hline Age at diagnosis (years) & 1,191 & $8.5(4.4)$ \\
\hline \multicolumn{3}{|l|}{ Age at visit (years) } \\
\hline $0-4$ & 15 & 1.3 \\
\hline $5-9$ & 240 & 20.2 \\
\hline $10-14$ & 407 & 34.2 \\
\hline$\geq 15$ & 529 & 44.4 \\
\hline Diabetes duration at visit (months) & 1,191 & $62.3(17.5)$ \\
\hline$\leq 36$ & 155 & 13.0 \\
\hline $37-48$ & 151 & 12.7 \\
\hline $49-60$ & 70 & 5.9 \\
\hline $60-72$ & 474 & 39.8 \\
\hline$>72$ & 341 & 28.6 \\
\hline \multicolumn{3}{|l|}{ Weight Status } \\
\hline Underweight/Normal Weight & 783 & 67.4 \\
\hline Overweight/Obese & 379 & 32.6 \\
\hline Missing & 29 & \\
\hline \multicolumn{3}{|l|}{ Insulin Regimen } \\
\hline Insulin Pump & 594 & 51.0 \\
\hline Glargine + rapid-acting insulin & 388 & 33.3 \\
\hline Glargine +2 or more insulins & 90 & 7.7 \\
\hline Multiple injections w/o Glargine & 48 & 4.1 \\
\hline Two or fewer insulin injections & 44 & 3.8 \\
\hline Missing & 27 & \\
\hline $\mathrm{A} 1 \mathrm{C}(\%)$ & 1,069 & $8.7(1.8)$ \\
\hline Triglycerides (mg/dL) & 974 & $83.3(56.3)$ \\
\hline LDL Cholesterol (mg/dL) & 977 & $94.7(28.3)$ \\
\hline
\end{tabular}




\begin{tabular}{|lcc|}
\hline Characteristic & $\mathbf{n}$ & $\boldsymbol{\%}^{\boldsymbol{a}}$ or mean $(\mathrm{SD})$ \\
HDL Cholesterol (mg/dL) & 977 & $57.5(14.0)$ \\
Total Cholesterol (mg/dL) & 977 & $169.1(34.7)$ \\
Systolic Blood Pressure (mm Hg) & 1160 & $104.9(11.2)$ \\
Diastolic Blood Pressure (mm Hg) & 1158 & $66.5(9.9)$ \\
\hline
\end{tabular}

${ }^{a}$ Percentages provided among individuals with non-missing data. 


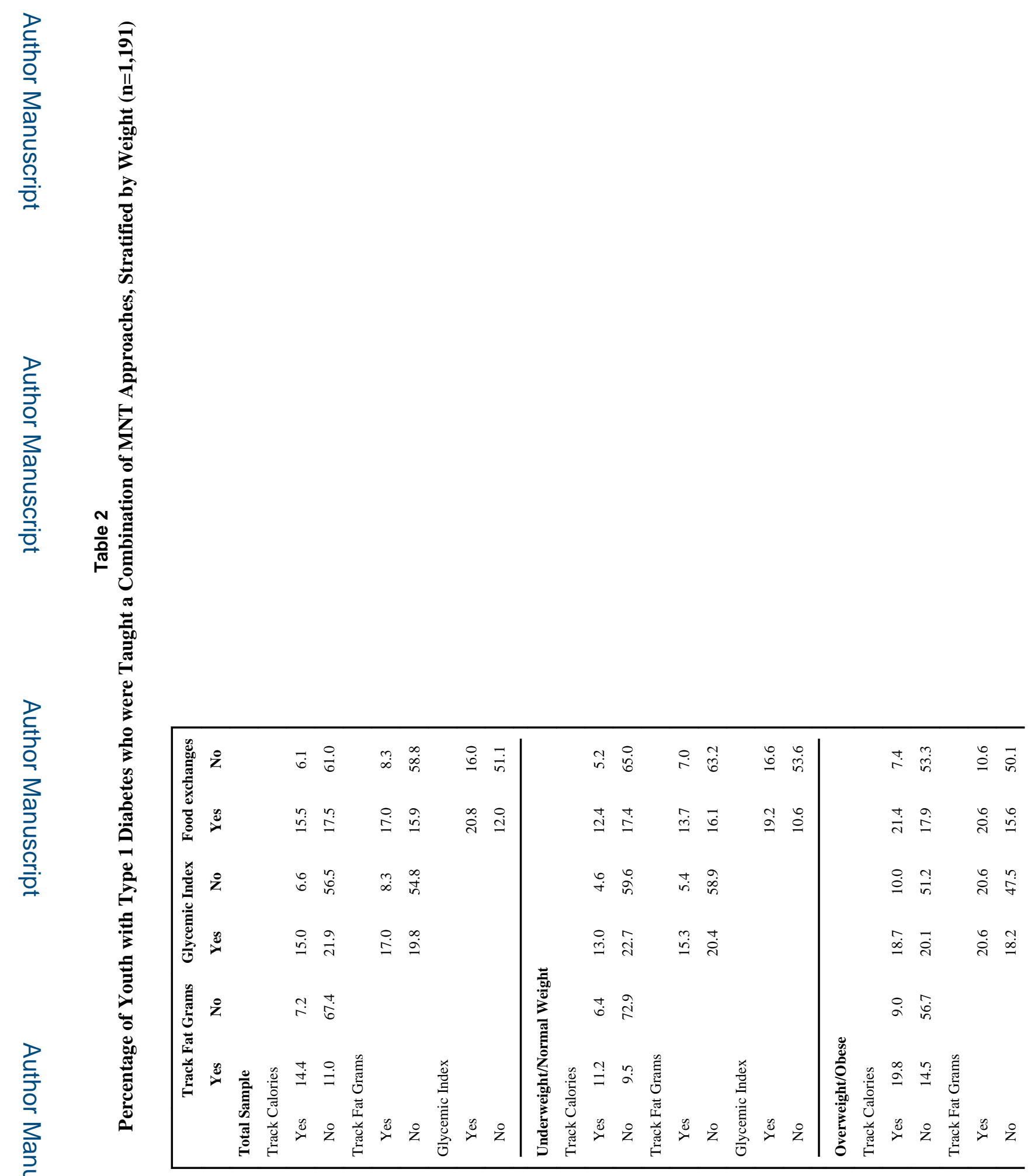

J Nutr Educ Behav. Author manuscript; available in PMC 2014 November 01. 


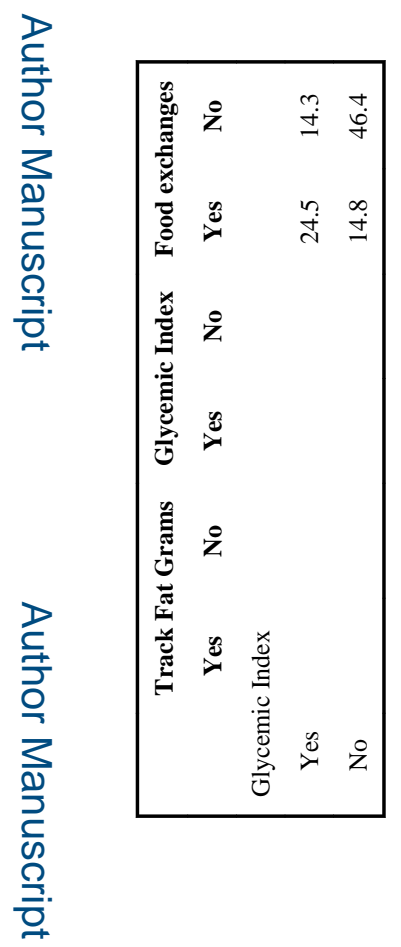


Table 3

Adjusted Associations ${ }^{a}$ of Frequency of Carbohydrate Counting Use with CVD Risk Factors among Participants who were Exclusively Taught Carbohydrate Counting, Stratified by Weight Status (n=499)

\begin{tabular}{|c|c|c|c|c|}
\hline & \multicolumn{2}{|c|}{$\begin{array}{c}\text { Underweight/Normal Weight Who Were Exclusively } \\
\text { Taught Carbohydrate Counting }\end{array}$} & \multicolumn{2}{|c|}{$\begin{array}{c}\text { Overweight/Obese Who Were Exclusively Taugh } \\
\text { Carbohydrate Counting }\end{array}$} \\
\hline & $\begin{array}{l}\text { Used Carbohydrate } \\
\text { Counting Often }\end{array}$ & $\begin{array}{c}\text { Used Carbohydrate } \\
\text { Counting Sometimes/ } \\
\text { Never }\end{array}$ & $\begin{array}{l}\text { Used Carbohydrate } \\
\text { Counting Often }\end{array}$ & $\begin{array}{c}\text { Used Carbohydrate } \\
\text { Counting Sometimes/ } \\
\text { Never }\end{array}$ \\
\hline LDL-Cholesterol (mg/dL) & $93.7(8.2)$ & $96.5(9.5)$ & $98.4(6.5)$ & $101.3(8.7)$ \\
\hline HDL-Cholesterol (mg/dL) & $63.2(4.3)$ & $60.5(5.0)$ & $58.8(3.9)$ & $58.9(5.2)$ \\
\hline Total Cholesterol (mg/dL) & $166.7(10.0)$ & $182(11.6)$ & $171.8(8.3)$ & $174.1(11.2)$ \\
\hline Triglycerides $(\mathrm{mg} / \mathrm{dL})^{*}$ & $56.3(12.1)$ & $98.6(14.1)$ & $72.5(14.9)$ & $69.1(20.0)$ \\
\hline $\mathrm{A} 1 \mathrm{c}(\%)^{*}$ & $8.3(0.4)$ & $9.4(0.4)$ & $8.4(0.4)$ & $8.3(0.5)$ \\
\hline SBP (mm Hg) & $106.0(2.6)$ & $106.2(3.05)$ & $106.5(2.3)$ & $100.9(2.9)$ \\
\hline DBP (mm Hg) & $65.8(2.2)$ & $69.4(2.6)$ & $65.4(2.2)$ & $63.5(2.7)$ \\
\hline
\end{tabular}

${ }^{a}$ Separate, multivariate linear regression models estimating mean value and standard errors for CVD risk factor by use of exclusive carbohydrate counting, adjusted for race/ethnicity, sex, parental education, age at visit, study site, diabetes duration, and insulin regimen stratified by weight. Referent category is "used carbohydrate counting often."

* For underweight/normal participants, significant difference between youth who used carbohydrate counting "sometimes/never" versus those who used the approach "often", p $<0.05$. 


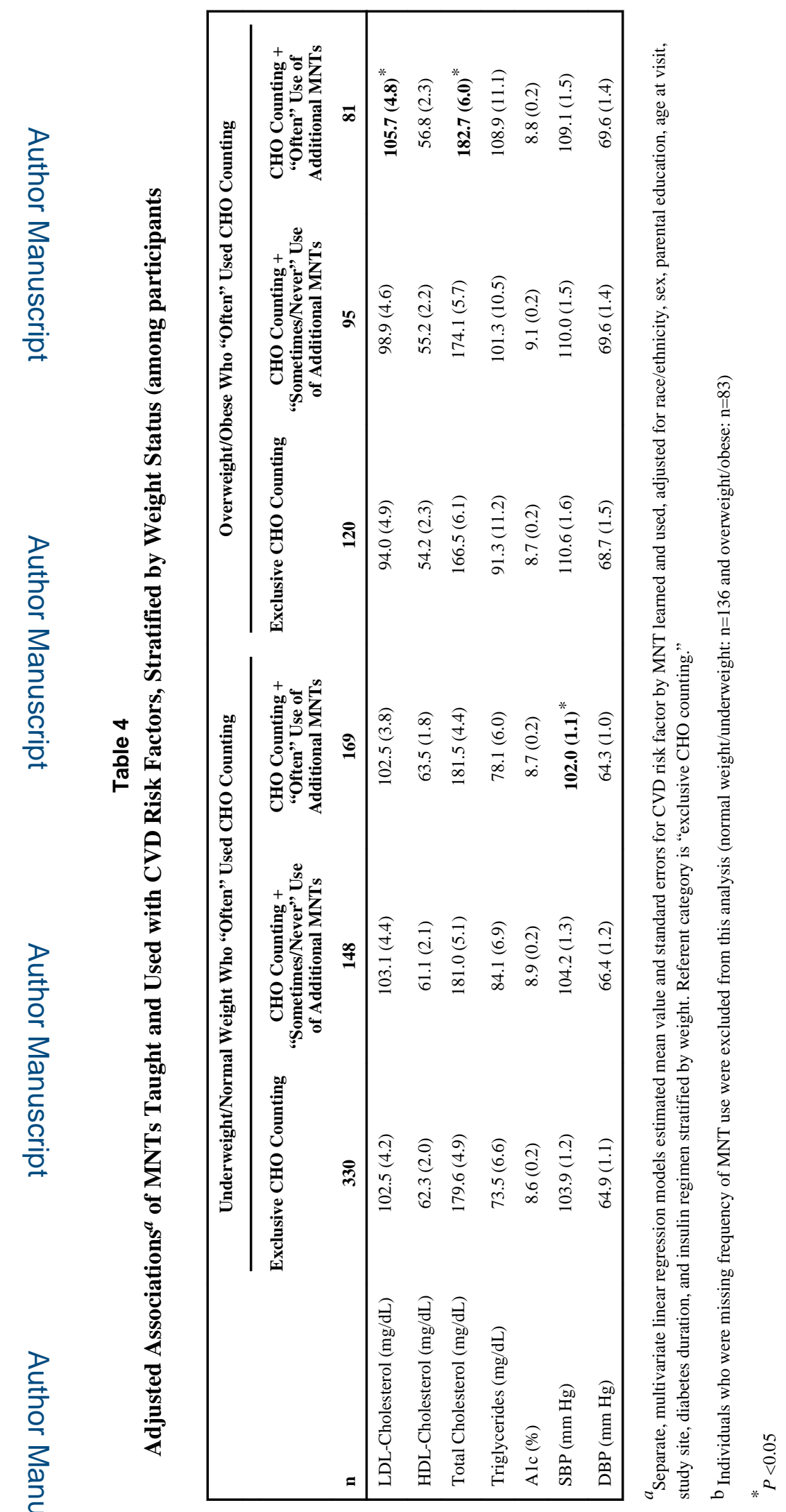

J Nutr Educ Behav. Author manuscript; available in PMC 2014 November 01. 